\title{
Diagnosis of Wind Energy System Faults Part I : Modeling of the Squirrel Cage Induction Generator
}

\author{
Lahcène Noureddine \\ Department of Electrical Engineering \\ Amar Thlidji University \\ Laghouat, Algeria
}

\author{
Omar Touhami \\ Research Laboratory of Electrotechnics \\ High National Polytechnic College \\ Algiers, Algeria
}

\begin{abstract}
Generating electrical power from wind energy is becoming increasingly important throughout the world. This fast development has attracted many researchers and electrical engineers to work on this field. The authors develop a dynamic model of the squirrel cage induction generator exists usually on wind energy systems, for the diagnosis of broken rotor bars defects from an approach of magnetically coupled multiple circuits. The generalized model is established on the base of mathematical recurrences. The winding function theory is used for determining the rotor resistances and the inductances in the case of $n$ - broken bars. Simulation results, in Part. II of this paper, confirm the validity of the proposed model.
\end{abstract}

Keywords-Induction Generator; Rotor Broken Bars; Faults Diagnosis; MCSA

\section{INTRODUCTION}

In a general way, the problem of fault diagnosis consists in the determination of the fault type with as much as possible details as the fault size, location and time of detection. From these important details, various approaches using mathematical models are developed for more than three decades, and it has a rich literature of hundreds of papers including many surveys $[1,2]$.

For the electrical machines, the faults, and their diagnosis techniques are reported in a great number of papers and categorized in three important sections:

Coupled magnetic circuit theory. This theory uses the finite element method associated the state space models, based on the Maxwell's equations by taking account of the machine geometry and the magnetic properties of materials. This method offers considerable advantages, such as the modeling of the topology of the coupled magnetic circuits and the capacity to model magnetic saturation on a high level of precision. However, the finite element method was not the privilege of all the researchers for the simulation of the rotor defects of the induction machines and remains today, little used because of the high computing time and the absence of on line knowledge of the faults [3, 4].

Multiple coupled circuit approach. The approach of the multiple coupled electric circuits is based on the Kirchhoff's laws. If in this method, magnetic saturation is neglected, it is however possible to observe the defects to the rotor and the stator, thanks to the design of a detailed model of an induction machine having m-stator circuits and nrotor bars, [5-7]. After having drawn up the equivalent scheme under operation normal conditions, it is possible then to write the equations of the meshed network and to simulate the faults in the rotor by the breaks of the rotor bars and/or an end ring segment.

Spectral analysis and parameter estimation. The procedure is based on the spectral analysis of the stator current signature and on the parameter estimation respectively, to identify the rotor bar defects. It is commonly called "Motor Current Signature Analysis.". The stator current spectra shows that the broken rotor bars give rise to a sequence of sidebands around the supply frequency $f$ [8]. These sidebands are at $f_{m}=f(1 \pm 2 k s), k=1,2 \ldots$ The lower sideband is specifically due to a broken bar, and the upper sideband is due to consequent speed oscillation. Another signal that has been proposed for motor fault analysis is an air-gap torque. Frequency components are observing at characteristic frequencies $f_{m}=f(2 k s)$. The fault spectra are dependent on the slip, which changes with the load.

The air-gap torque spectrum and the current stator spectrum are a potential signature that can be used by on line fault diagnosis. The analytical knowledge in the process is also used to produce quantifiable, analytical information. The first characteristic values are generated by the process analysis using mathematical process models together with parameter estimation, state estimation and parity equation methods. Characteristic values are parameters, state variables or residuals. As an example: Broken bar detection on an induction machine using state and parameter estimation techniques have been reported in $[9,10]$.

This paper presents a generalized induction machine model for the diagnosis of rotor defects by an approach of magnetically coupled multiple circuits (using ideas A.R. Muñoz and T. A. Lip, [11]). The developed inductances (self and mutual inductances) were given for rotors having an unspecified number of broken rotor bars, by using of mathematical recurrences. Considering the configuration of the rotor, it is more realistic to model the cage as $\mathrm{m}$ identical magnetically coupled circuits. Moreover, one advantage of this approach is that it is applicable for all numbers of broken bars per pole pair. From where the need for determining inductances and resistances to the rotor meshes affected by the defect. Experimental results and simulation in Part. II of this paper confirm the validity of the proposed approach. 


\section{Modeling OF SCIG BY A MultiPle Coupled CiRCUIT APPROACH}

The three-phase model does not make it possible to know the actual values of the currents circulating in the rotor bars and thus does not allow determining the defects with the rotor. This is why the model multi-windings were developing. One will represent then, a model of the rotor as being made up with as many phases as bars; what results in regarding the currents circulating in the end ring segments as rotor phase currents $[12,13]$.

By taking account of the following assumptions:

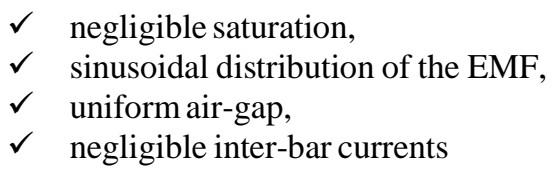

\section{A. Stator equations}

The stator equations are written by:

$$
v_{s}=R_{s} \cdot i_{s}+\frac{d \phi_{s}}{d t}
$$

With: $v_{s}=\left[\begin{array}{lll}v_{a} & v_{b} & v_{c}\end{array}\right]^{T}, i_{s}=\left[\begin{array}{lll}i_{a} & i_{b} & i_{c}\end{array}\right]^{T}$

The resistance matrix (diagonal $(3 \times 3)$ ) contains resistance of each winding:

$$
R_{s}=\left[\begin{array}{ccc}
r_{s} & 0 & 0 \\
0 & r_{s} & 0 \\
0 & 0 & r_{s}
\end{array}\right]
$$

And the total stator flux is:

$$
\begin{aligned}
& \phi_{s}=\phi_{s s}+\phi_{s r} \\
& \phi_{s}=L_{s} i_{s}+L_{s r} i_{r}
\end{aligned}
$$

Where: $\phi_{s s}$ is stator flux due to the stator currents and $\phi_{s r}$ is stator flux due to the rotor currents.

With:

$$
L_{s}=\left[\begin{array}{ccc}
L_{l s}+L_{m s} & -\frac{L_{m s}}{2} & -\frac{L_{m s}}{2} \\
-\frac{L_{m s}}{2} & L_{l s}+L_{m s} & -\frac{L_{m s}}{2} \\
-\frac{L_{m s}}{2} & -\frac{L_{m s}}{2} & L_{l s}+L_{m s}
\end{array}\right]
$$

Where $L_{l s}$ and $L_{m s}$ are respectively leakage and magnetizing inductances of stator windings. Magnetizing inductance $L_{m s}$ for a winding having $N_{s}$ number of stator phase turns in series, is given by:

$$
L_{m s}=\frac{\mu_{0} l r}{g} N_{s}^{2}\left(\frac{\pi}{4}\right)
$$

Leakage inductance is computed by [11].
Each rotor mesh is made of two adjacent bars and the end- ring segment, which connect them and is magnetically coupled with all the other rotor meshes and the three-phase stator. Mutual inductance matrix $\left(3 \mathrm{x} N_{r}\right)$ stator-rotor is:

$$
L_{s r}=\left[\begin{array}{cccc}
L_{a 1} & L_{a 2} & \ldots & L_{a N_{r}} \\
L_{b 1} & L_{b 2} & \ldots & L_{b N_{r}} \\
L_{c 1} & L_{c 2} & \ldots & L_{c N_{r}}
\end{array}\right]
$$

The inductances are computed by using winding function theory "WFT". This method supposes that there is no symmetry according to the winding function theory [6]. Mutual inductance between two unspecified reels " $i$ " and " $j "$ of the machine can be calculated from Eq.(5), by supposing that iron permeance is infinite [14].

$$
L_{i j}(\theta)=\mu_{0} l r \int_{0}^{2 \pi} g^{-1}(\varphi, \theta) N_{i}(\varphi, \theta) N_{j}(\varphi, \theta) d \varphi
$$

Where $\theta$ represents the rotor position compared to a given reference (related to the stator). $\varphi$ is a particular angular position along the interior surface of the stator. $g^{-1}(\varphi$, $\theta)$ is the inverse function of the air-gap, if we then suppose that the air-gap is constant and small compared to rotor ray, the function is equal to $1 / g$. The term $N i(\varphi, \theta)$ is known as winding function and represent the spatial distribution of the MMF along the air-gap for a unit current circulating in winding " $i$ ". Leakage inductances are calculated by only setting $i=j$. From winding function theory, one can determine the MMF of air-gap produced by a current $i a$ circulating in winding " $a$ " for that it position of the air-gap, as follows:

$F_{a}=N_{a}(\theta) \cdot i_{a}$

The created flux in a reel, having $N_{b}$ whorls, by the current $i_{a}$ is given by:

$\phi=F . \lambda$

Where $\lambda$ and $\mathrm{F}$ are respectively the permeance and air-gap magneto-motive force. The differential flux across the air-gap

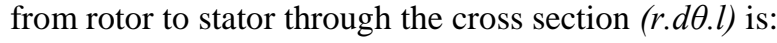

$d \phi=F_{a}(\theta) \cdot \mu_{0} \cdot l \cdot r \cdot d \theta / g$

The differential flux created in the reel $B$ is:

$d \Lambda_{b a}=N_{a}(\theta) \cdot N_{b}(\theta) i_{a}(\theta) \cdot \mu_{0} \cdot$ l.r. $\cdot d \theta / g$

From where the total fluxes expression:

$$
\Lambda_{b a}=\mu_{0} \cdot l \cdot r \cdot i_{a} \cdot g^{-1} \int_{\theta_{b 1}}^{\theta_{b 2}} N_{a}(\theta) \cdot N_{b}(\theta) \cdot d \theta
$$

From total flux, it is then possible to determine inductance between reel $a$ and reel $b$ : 
$L_{b a}=\frac{\mu_{0} \cdot l \cdot r}{g} \int_{\theta_{b 1}}^{\theta_{b 2}} N_{a}(\theta) \cdot N_{b}(\theta) \cdot d \theta$

By considering the distribution of the stator windings and the rotor bars, the winding function for the stator phases is:

$N_{a}=\frac{N_{s}}{2} \cos (\theta)$

$N_{b}=\frac{N_{s}}{2} \cos \left(\theta-\frac{2 \pi}{3}\right)$

$N_{c}=\frac{N_{s}}{2} \cos \left(\theta+\frac{2 \pi}{3}\right)$

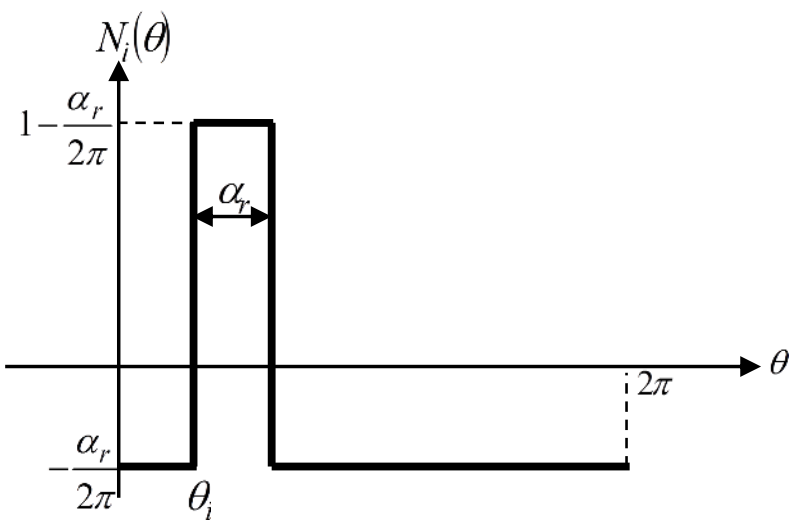

Fig. 1. Rotor loop winding function of healthy generator

The winding function for the $i^{\text {th }}$ mesh of the rotor is:

$N_{i}=\left\{\begin{array}{cc}-\alpha_{r} / 2 \pi & 0<\theta \leq \theta_{i} \\ 1-\alpha_{r} / 2 \pi & \theta_{i}<\theta \leq \theta_{i+1} \\ -\alpha_{r} / 2 \pi & \theta_{i+1}<\theta \leq 2 \pi\end{array}\right.$

Where $\alpha_{r}$ is the angle between two adjacent bars and $\theta_{i}$ the angle of the bar $i^{\text {th }}$. By replacing $g^{-1}(\varphi, \theta)$ and $N(\varphi, \theta)$ previously defined in the expression (7), for the phase " $a$ ", one will determine following inductances:

$L_{a i}=\frac{\mu_{0} l r}{g} \int_{0}^{2 \pi} N_{a}(\theta) N_{i}(\theta) d \theta=$

$\frac{\mu_{0} l r N_{s}}{2 g}\left(-\int_{0}^{\theta_{i}} \frac{\alpha_{r}}{2 \pi} \cos \theta d \theta+\int_{\theta_{i}}^{\theta_{i+1}}\left(1-\frac{\alpha_{r}}{2 \pi}\right) \cos \theta d \theta-\int_{\theta_{i+1}}^{2 \pi} \frac{\alpha_{r}}{2 \pi} \cos \theta d \theta\right)$

After integration this expression yields

$L_{a i}=\frac{\mu_{0} l r}{g} \frac{N_{s}}{2}\left(\sin \theta_{i+1}-\sin \theta_{i}\right)$

$\theta_{i}$ can be set in the following form: $\theta_{i}=\theta_{r}+(i-1) \alpha_{r}$, with $\theta_{r}$ is an arbitrary angle.

so :

$$
L_{a i}=\frac{\mu_{0} l r}{g} \frac{N_{s}}{2}\left[\sin \left(\theta_{r}+i \alpha_{r}\right)-\sin \left(\theta_{r}+(i-1) \alpha_{r}\right)\right]
$$

After transformation by trigonometric relations:

$L_{a i}=\frac{\mu_{0} l r N_{s}}{g} \sin \frac{\alpha_{r}}{2} \cos \left(\theta_{r}+\left(i-\frac{1}{2}\right) \alpha_{r}\right)$

Let:

$L_{m}=\frac{\mu_{0} l r N_{s}}{g} \quad$ with : $\delta=\frac{\alpha_{r}}{2}$

We obtain:

$L_{a i}=L_{m} \sin \delta \cos \left(\theta_{i}+\delta\right)$

It is then easy to find mutual inductances for two other phases.

\section{B. Rotor equations}

For needs for simple comprehension, the rotor cage is modeled by an equivalent circuit containing $k+1$ magnetically meshes. The rotor bars are numbered from 1 to $k+1$.

It is to be considered that each mesh is defined by two adjacent bars of the rotor and connected between them by endring segment. Moreover, each rotor bar and end ring segment are replaced by an equivalent circuit represented by a resistance and an inductance, as the Fig. 2 shows it.

From these equivalent circuits of a squirrel cage rotor, one can write the following equations:

$v_{r}=R_{r} \cdot i_{r}+\frac{d \phi_{r}}{d t}$

With :

$\phi_{r}=L_{s r}^{T} i_{s}+L_{r} i_{r}$

$v_{r}=\left[\begin{array}{lllllll}v_{r 1} & v_{r 2} & \ldots & v_{r k} & \ldots & v_{r N_{r}} & v_{e}\end{array}\right]^{T}=O$

$i_{r}=\left[\begin{array}{lllllll}i_{r 1} & i_{r 2} & \ldots & i_{r k} & \ldots & i_{r N_{r}} & i_{e}\end{array}\right]^{T}$

$\phi_{r}=\left[\begin{array}{lllllll}\phi_{r 1} & \phi_{r 2} & \ldots & \phi_{r k} & \ldots & \phi_{r N_{r}} & \phi_{e}\end{array}\right]^{T}$

The rotor resistance matrix $\left(N_{r} \mathrm{x} N_{r}\right)$ is:

$R_{r}=\left[\begin{array}{cccccc}R_{0} & -R_{b} & 0 & \ldots & 0 & -R_{b} \\ -R_{b} & R_{0} & -R_{b} & \ldots & . & 0 \\ 0 & -R_{b} & R_{0} & \ldots & . & . \\ . & . & . & \ldots & . & . \\ \cdot & . & . & \ldots & R_{0} & -R_{b} \\ -R_{b} & . & . & \ldots & -R_{b} & R_{0}\end{array}\right]$

With :

$R_{0}=2\left(R_{b}+R_{e}\right)$

$L_{0}=L_{m r}+2\left(L_{b}+L_{e}\right)$ 


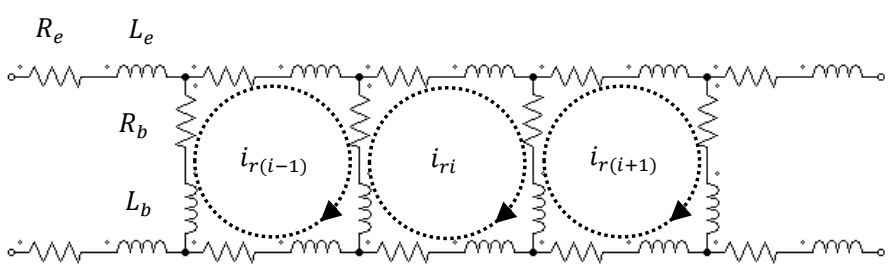

Fig. 2. Equivalent circuits of a squirrel cage rotor for Healthy induction generator

Self-inductance of the meshes is equal, and they are given by:

$L_{m r}=\frac{\mu_{0} l r}{g} \int_{0}^{2 \pi} N_{i}^{2}(\theta) d \theta=$

$\frac{\mu_{0} l r}{g}\left(\int_{0}^{\theta_{i}}\left(-\frac{\alpha_{r}}{2 \pi}\right)^{2} d \theta+\int_{\theta_{i}}^{\theta_{i+1}}\left(1-\frac{\alpha_{r}}{2 \pi}\right)^{2} d \theta+\int_{\theta_{i+1}}^{2 \pi}\left(-\frac{\alpha_{r}}{2 \pi}\right)^{2} d \theta\right)$

$=\frac{\mu_{0} l r}{g} \alpha_{r}\left(1-\frac{\alpha_{r}}{2 \pi}\right)$

Rotor-rotor mutual inductance between two rotor meshes is deduced from Eq.(19)
$L_{k i}=\frac{\mu_{0} l r}{g} \int_{0}^{2 \pi} N_{k}(\theta) N_{i}(\theta) d \theta=-\frac{\mu_{0} l r}{g}\left(\frac{\alpha_{r}^{2}}{2 \pi}\right)$

Electromagnetic torque produced by the machine can be expressed from the magnetic co-energy $W_{c o}$ as,

$$
\Gamma_{e}=\left[\frac{\partial W_{c o}}{\partial \theta}\right]_{\left(i_{s}, i_{r} \text { constant }\right)}
$$

It is previously defined that inductances $L_{s}$ and $L_{r}$ contain only constant elements and the electromagnetic torque is a scalar quantity. The final expression of the torque is thus reduced to

$$
\Gamma_{e}=\frac{1}{2} P i_{s}{ }^{T} \frac{\partial L_{s r}\left(\theta_{r}\right)}{\partial \theta_{r}} i_{r}
$$

Where $P$ is the number of pole pairs and $\theta_{r}$ is the rotor displacement in electrical radians.

$$
L_{r}=\left[\begin{array}{cccccc}
L_{0} & L_{12}-L_{b} & L_{13} & \ldots & L_{1\left(N_{r}-1\right)} & L_{1 N_{r}}-L_{b} \\
L_{21}-L_{b} & L_{0} & L_{23}-L_{b} & \ldots & . & L_{2 N_{r}} \\
L_{31} & L_{32}-L_{b} & . & \ldots & . & . \\
\vdots & . & . & \ldots & . & . \\
\vdots & . & . & \ldots & . & L_{\left(N_{r}-1\right) N_{r}}-L_{b} \\
L_{N_{r} 1}-L_{b} & . & . & \ldots & L_{N_{r}\left(N_{r}-1\right)}-L_{b} & L_{0}
\end{array}\right]
$$$$
\Gamma_{e}=\frac{P}{2} L_{m}\left\{\left(i_{a}-\frac{1}{2} i_{b}-\frac{1}{2} i_{c}\right) \sum_{k=1}^{N_{r}} i_{r k} \sin \left(\theta_{r}+(k-1) \alpha_{r}\right)+\frac{\sqrt{3}}{2}\left(i_{c}-i_{b}\right) \sum_{k=1}^{N_{r}} i_{r k} \cos \left(\theta_{r}+(k-1) \alpha_{r}\right)\right\}
$$

\section{Broken rotor bar faults}

1) Generator with one broken bar

The rupture of a bar decreases the number of equations to the rotor of 1 , because the defect imposes the following condition:

If it is the bar traversed by $i_{r i}$ and $i_{r(i+1)}$ which is broken, one has $i_{r i}=i_{r(i+l)}$ which wants to say that current $i_{r i}$ traverses a mesh twice broader and the mesh $i+1$ is eliminated, Fig.(3).

In the inductance matrix the line and the column $i+1$ are eliminated and the terms relating to the column $i$ are thus recomputed by using the expression (5) by taking account of the new function of winding for the rotor mesh $i$,

$$
N_{i}=\left\{\begin{array}{cc}
-\alpha_{r} / 2 \pi & 0<\theta \leq \theta_{i} \\
1-\alpha_{r} / 2 \pi & \theta_{i}<\theta \leq \theta_{i+2} \\
-\alpha_{r} / 2 \pi & \theta_{i+2}<\theta \leq 2 \pi
\end{array}\right.
$$

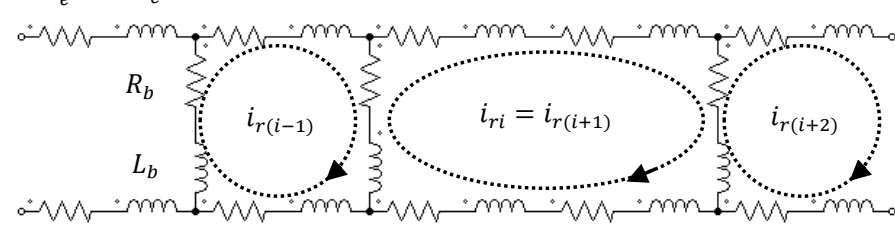

Fig. 3. Equivalent circuits of a squirrel cage rotor with one broken bar

Calculation of new mutual inductance $L_{a i}$ between the mesh $i$ and stator winding $a$ :

$$
L_{a i}=\frac{\mu_{0} l r}{g} \int_{0}^{2 \pi} N_{a}(\theta) N_{i}(\theta) d \theta=L_{m} \sin (2 \delta) \cos \left(\theta_{i}+2 \delta\right)
$$

The mutual inductance matrix stator rotor $\left(3 \mathrm{x}\left(N_{r^{-}}-1\right)\right)$ becomes:

$$
\left[L_{s r}\right]=\left[\begin{array}{ccccccc}
L_{a 1} & L_{a 2} & \ldots & L_{a i} & L_{a(i+2)} & \ldots & L_{a N_{r}} \\
L_{b 1} & L_{b 2} & \ldots & L_{b i} & L_{b(i+2)} & \ldots & L_{b N_{r}} \\
L_{c 1} & L_{c 2} & \ldots & L_{c i} & L_{c(i+2)} & \ldots & L_{c N_{r}}
\end{array}\right]
$$


The same calculation is applied for the $i^{\text {th }}$ line, the new matrix inductance of rotor is $\left(N_{r}-1\right) \mathrm{x}\left(N_{r}-1\right)$ becomes: $L_{r}=\left[\begin{array}{cccccc}L_{0} & L_{12}-L_{b} & L_{13} & \ldots & L_{1\left(N_{r}-1\right)} & L_{1 N_{r}}-L_{b} \\ L_{21}-L_{b} & L_{0} & L_{23}-L_{b} & \ldots & . & L_{2 N_{r}} \\ L_{31} & L_{32}-L_{b} & . & \ldots & . & . \\ \vdots & \vdots & \vdots & L_{0 i} & \vdots & \vdots \\ \vdots & . & . & \ldots & L_{0} & L_{\left(N_{r}-1\right) N_{r}}-L_{b} \\ L_{N_{r} 1}-L_{b} & . & . & \ldots & L_{N_{r}\left(N_{r}-1\right)}-L_{b} & L_{0}\end{array}\right]$

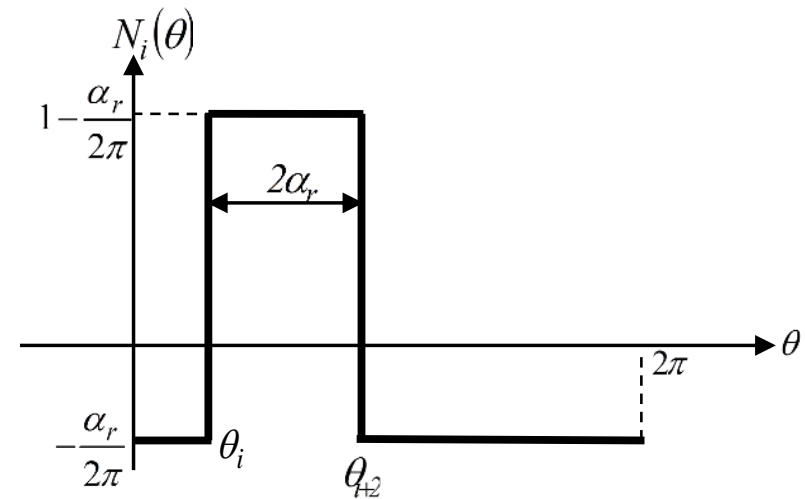

Fig. 4. Rotor loop winding function of faulty generator (1bb)

The rotor resistance matrix is affected by the break of the bar, and is obtained from the new representation $\left(N_{r^{-}}-1\right) \times\left(N_{r^{-}}-1\right)$ of the rotor:

$R_{r}=\left[\begin{array}{cccccc}R_{0} & -R_{b} & \cdots & 0 & \cdots & -R_{b} \\ -R_{b} & R_{0} & \cdots & \cdots & \cdot & 0 \\ \vdots & \vdots & \cdots & \vdots & \cdot & \cdot \\ \cdot & \cdot & \cdot & R_{0 i} & \cdot & \cdot \\ \cdot & \cdot & \cdot & \vdots & \vdots & \vdots \\ -R_{b} & \cdot & \cdot & \cdots & \cdots & R_{0}\end{array}\right]$

With:

$R_{0 i}=2\left(R_{b}+2 R_{e}\right)$

Determination of the new self-inductance $L_{0 i}$ of the rotor:

$L_{0 i}=L_{i i}+2\left(L_{b}+2 L_{e}\right)$

The self-inductance of the $i^{\text {th }}$ rotor mesh becomes:

$L_{i i}=\frac{\mu_{0} l r}{g} \int_{0}^{2 \pi} N_{i}^{2}(\theta) d \theta=\frac{\mu_{0} l r}{g} \alpha_{r}\left(2-3 \frac{\alpha_{r}}{2 \pi}\right)$

The mutual inductance between the $k^{\text {th }}$ mesh and the $i^{\text {th }}$ mesh of the rotor is recalculated as follows:

$L_{k i}=\frac{\mu_{0} l r}{g} \int_{0}^{2 \pi} N_{k}(\theta) N_{i}(\theta) d \theta=-2 \frac{\mu_{0} l r}{g}\left(\frac{\alpha_{r}^{2}}{2 \pi}\right)$

2) Generator with two adjacent broken bars

In this case, the number of rotor meshes falls by 2 , if the first bar traversed by the currents $i_{r i}, i_{r(i+l)}$ and second bar traversed by the currents $i_{r(i+1)}$ and $i_{r(i+2)}$ are broken, one have $i_{r i}=i_{r(i+1)}=i_{r(i+2)}$ means that $i_{r i}$ traverses a mesh three times broader as shows it in Fig.(5). At the time of the break of two bars the function of winding becomes:

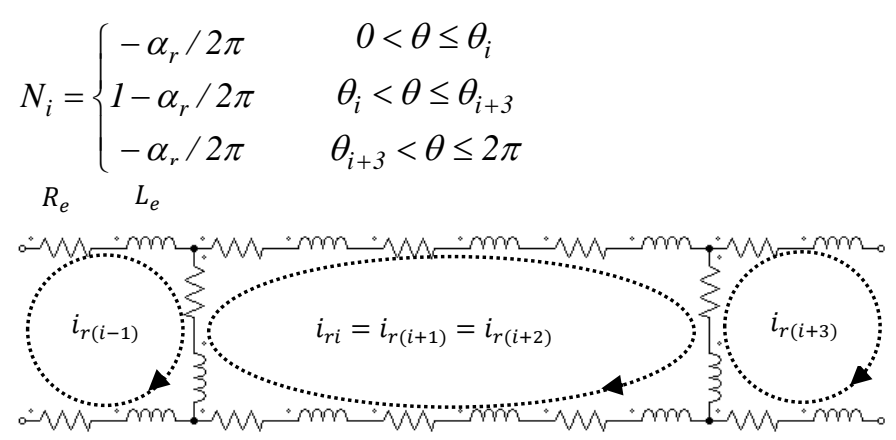

Fig. 5. Equivalent circuits of a squirrel cage rotor with two broken bars

The same type of relation applied in the case of a broken bar is used for the calculation of the new inductance and resistance matrices.

The mutual inductance matrix stator rotor $\left(3 \mathrm{x}\left(N_{r}-2\right)\right)$ becomes:

$\left[L_{s r}\right]=\left[\begin{array}{ccccccc}L_{a 1} & L_{a 2} & \ldots & L_{a i} & L_{a(i+3)} & \ldots & L_{a N_{r}} \\ L_{b 1} & L_{b 2} & \ldots & L_{b i} & L_{b(i+3)} & \ldots & L_{b N_{r}} \\ L_{c 1} & L_{c 2} & \ldots & L_{c i} & L_{c(i+3)} & \ldots & L_{c N_{r}}\end{array}\right]$

The same calculation is applied for the $i^{\text {th }}$ line, the new matrix inductance of rotor is $\left(N_{r}-2\right) \times\left(N_{r}-2\right)$ dimensions, with:

$L_{0 i}=L_{i i}+2\left(L_{b}+3 L_{e}\right)$

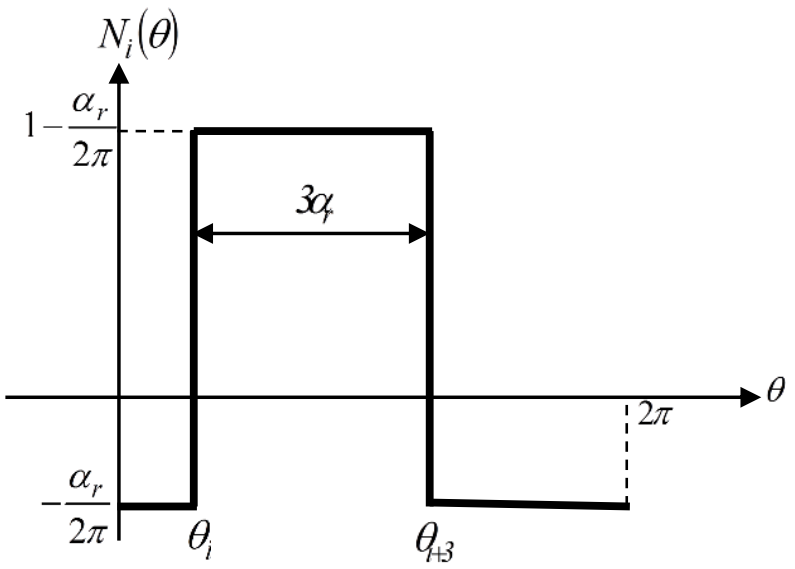

Fig. 6. Rotor loop winding function of faulty generator (2 adjacent bb) 
And the self-inductance of winding $i$ is

$L_{i i}=\frac{\mu_{0} l r}{g} \int_{0}^{2 \pi} N_{i}^{2}(\theta) d \theta=\frac{\mu_{0} l r}{g} \alpha_{r}\left(3-5 \frac{\alpha_{r}}{2 \pi}\right)$

The mutual inductance between the $k^{\text {th }}$ mesh and the $i^{\text {th }}$ mesh of the rotor is recalculated as follows:

$L_{k i}=\frac{\mu_{0} l r}{g} \int_{0}^{2 \pi} N_{k}(\theta) N_{i}(\theta) d \theta=-3 \frac{\mu_{0} l r}{g}\left(\frac{\alpha_{r}^{2}}{2 \pi}\right)$

Determination of mutual inductances

$L_{a i}=\frac{\mu_{0} l r}{g} \int_{0}^{2 \pi} N_{a}(\theta) N_{i}(\theta) d \theta=L_{m} \sin (3 \delta) \cos \left(\theta_{i}+3 \delta\right)$

The rotor resistance matrix is affected by the break of the bar, and is obtained from the new representation $\left(N_{r}-2\right) \mathrm{x}\left(N_{r}-2\right)$ of the rotor, with :

$R_{0 i}=2\left(R_{b}+3 R_{e}\right)$

3) Generalized model by mathematical recurrences

The same reasoning, that in the case of a bar and two broken bars, is valid in the case where there are several broken successive adjacent bars. By mathematical recurrences, we determine the generalized model. The number of rotor equations will be fallen according to the number of broken bars, and the meshes concerned with the break are eliminated; the mesh $i$ will be $n$ time broader.

The mutual inductance matrix stator rotor $\left(3 \mathrm{x}\left(N_{r}-n\right)\right)$ becomes:

$\left[L_{s r}\right]=\left[\begin{array}{ccccccc}L_{a 1} & L_{a 2} & \ldots & L_{a i} & L_{a(i+(n+1))} & \ldots & L_{a N_{r}} \\ L_{b 1} & L_{b 2} & \ldots & L_{b i} & L_{b(i+(n+1))} & \ldots & L_{b N_{r}} \\ L_{c 1} & L_{c 2} & \ldots & L_{c i} & L_{c(i+(n+1))} & \ldots & L_{c N_{r}}\end{array}\right]$

The same calculation is applied for the $i^{\text {th }}$ line, the new matrix inductance of rotor is $\left(N_{r}-n\right) \times\left(N_{r}-n\right)$ dimensions, with:

$L_{0 i}=L_{i i}+2\left(L_{b}+(n+1) L_{e}\right)$

And the self-inductance of winding $I$ is

$L_{i i}=\frac{\mu_{0} l r}{g} \int_{0}^{2 \pi} N_{i}^{2}(\theta) d \theta=\frac{\mu_{0} l r}{g} \alpha_{r}\left((n+1)-(2 n+1) \frac{\alpha_{r}}{2 \pi}\right)$

The mutual inductance between the $k^{\text {th }}$ mesh and the $i^{\text {th }}$ mesh of the rotor is recalculated as follows:

$L_{k i}=\frac{\mu_{0} l r}{g} \int_{0}^{2 \pi} N_{k}(\theta) N_{i}(\theta) d \theta=-(n+1) \frac{\mu_{0} l r}{g}\left(\frac{\alpha_{r}^{2}}{2 \pi}\right)$

Determination of mutual inductances

$L_{a i}=\frac{\mu_{0} l r}{g} \int_{0}^{2 \pi} N_{a}(\theta) N_{i}(\theta) d \theta=L_{m} \sin ((n+1) \delta) \cos \left(\theta_{i}+(n+1) \delta\right)$

The rotor resistance matrix becomes $\left(N_{r}-n\right) \times\left(N_{r}-n\right)$ with:

$R_{0 i}=2\left(R_{b}+(n+1) R_{e}\right)$
4) Generator with two non-adjacent broken bars

Now we suppose that the two broken bars are not adjacent, it is obvious that the matrices resistances and inductances of the previous section are not the same. We will see that there is a new mutual inductance between the two cells and involved two broken non adjacent bars as shown in the following figure:

$$
R_{e} \quad L_{e}
$$

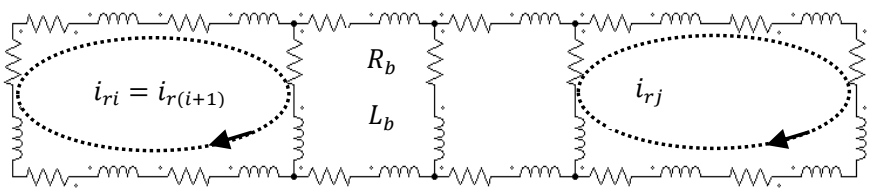

Fig. 7. Equivalent circuits of a squirrel cage rotor with two non-adjacent broken bars

This failure reduces the number of rotor equations with two equations, but one will have:

$i_{r i}=i_{r(i+1)}$

$i_{r j}=i_{r(j+1)}$

The mutual inductances (stator-rotor, rotor-rotor) and the self-inductance of the mesh $i r$ remains the same as we saw in the first part. With the same procedure we can calculate the inductances of the mesh $j r$, such as:

$N_{j}=\left\{\begin{array}{cc}-\alpha_{r} / 2 \pi & 0<\theta \leq \theta_{j} \\ 1-\alpha_{r} / 2 \pi & \theta_{j}<\theta \leq \theta_{j+2} \\ -\alpha_{r} / 2 \pi & \theta_{j+2}<\theta \leq 2 \pi\end{array}\right.$

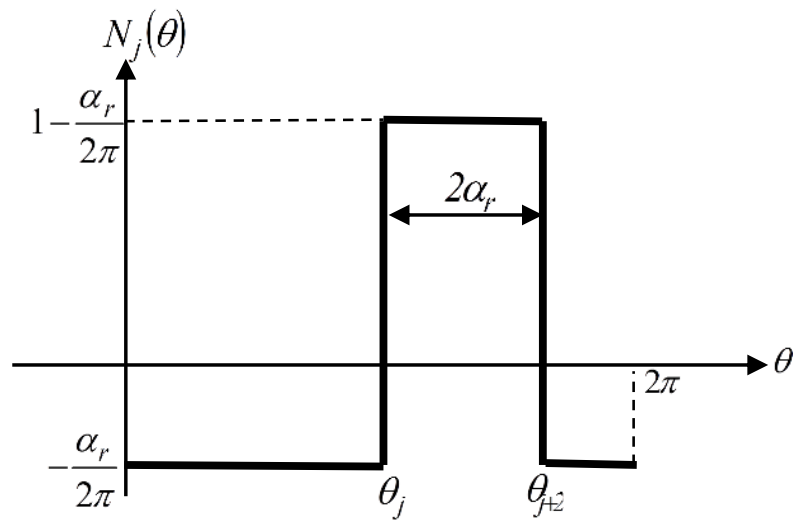

Fig. 8. Rotor loop winding function of faulty generator (2 non-adjacent bb)

Calculation of stator-rotor mutual inductances of the two mesh $i_{r}$ and $j_{r}$ :

$$
\begin{aligned}
& L_{a i}=\frac{\mu_{0} l r}{g} \int_{0}^{2 \pi} N_{a}(\theta) N_{i}(\theta) d \theta=\frac{\mu_{0} l r}{g} N_{s} \sin (2 \delta) \cos \left(\theta_{i}+2 \delta\right) \\
& L_{a j}=\frac{\mu_{0} l r}{g} \int_{0}^{2 \pi} N_{a}(\theta) N_{j}(\theta) d \theta=\frac{\mu_{0} l r}{g} N_{s} \sin (2 \delta) \cos \left(\theta_{j}+2 \delta\right)
\end{aligned}
$$

The matrix of the stator-rotor mutual inductance becomes: 


$$
\begin{gathered}
{\left[L_{s r}\right]=\left[\begin{array}{cccccccccc}
L_{a 1} & L_{a 2} & \ldots & L_{a i} & L_{a(i+2)} & \ldots & L_{a j} & L_{a(j+2)} & \ldots & L_{a N_{r}} \\
L_{b 1} & L_{b 2} & \ldots & L_{b i} & L_{b(i+2)} & \ldots & L_{b j} & L_{b(j+2)} & \ldots & L_{b N_{r}} \\
L_{c 1} & L_{c 2} & \ldots & L_{c i} & L_{c(i+2)} & \ldots & L_{c j} & L_{c(j+2)} & \ldots & L_{c N_{r}}
\end{array}\right] \quad L_{k i}=\frac{\mu_{0} l r}{g} \int_{0}^{2 \pi} N_{k}(\theta) N_{i}(\theta) d \theta=-2 \frac{\mu_{0} l r}{g}\left(\frac{\alpha_{r}^{2}}{2 \pi}\right)} \\
L_{k j}=\frac{\mu_{0} l r}{g} \int_{0}^{2 \pi} N_{k}(\theta) N_{j}(\theta) d \theta=-2 \frac{\mu_{0} l r}{g}\left(\frac{\alpha_{r}^{2}}{2 \pi}\right)
\end{gathered}
$$

$L_{i i}=\frac{\mu_{0} l r}{g} \int_{0}^{2 \pi} N_{i}^{2}(\theta) d \theta=\frac{\mu_{0} l r}{g} \alpha_{r}\left(2-3 \frac{\alpha_{r}}{2 \pi}\right)$

$L_{j j}=\frac{\mu_{0} l r}{g} \int_{0}^{2 \pi} N_{j}^{2}(\theta) d \theta=\frac{\mu_{0} l r}{g} \alpha_{r}\left(2-3 \frac{\alpha_{r}}{2 \pi}\right)$ meshes:

$L_{i j}=\frac{\mu_{0} l r}{g} \int_{0}^{2 \pi} N_{i}(\theta) N_{j}(\theta) d \theta=-3 \frac{\mu_{0} l r}{g}\left(\frac{\alpha_{r}^{2}}{2 \pi}\right)$

The matrix of the rotor inductances becomes:

Calculation of mutual inductances between $k^{\text {th }}$ and $i^{\text {th }}$ meshes firstly, on the other hand between $k^{\text {th }}$ and $j^{\text {th }}$ meshes of rotor :

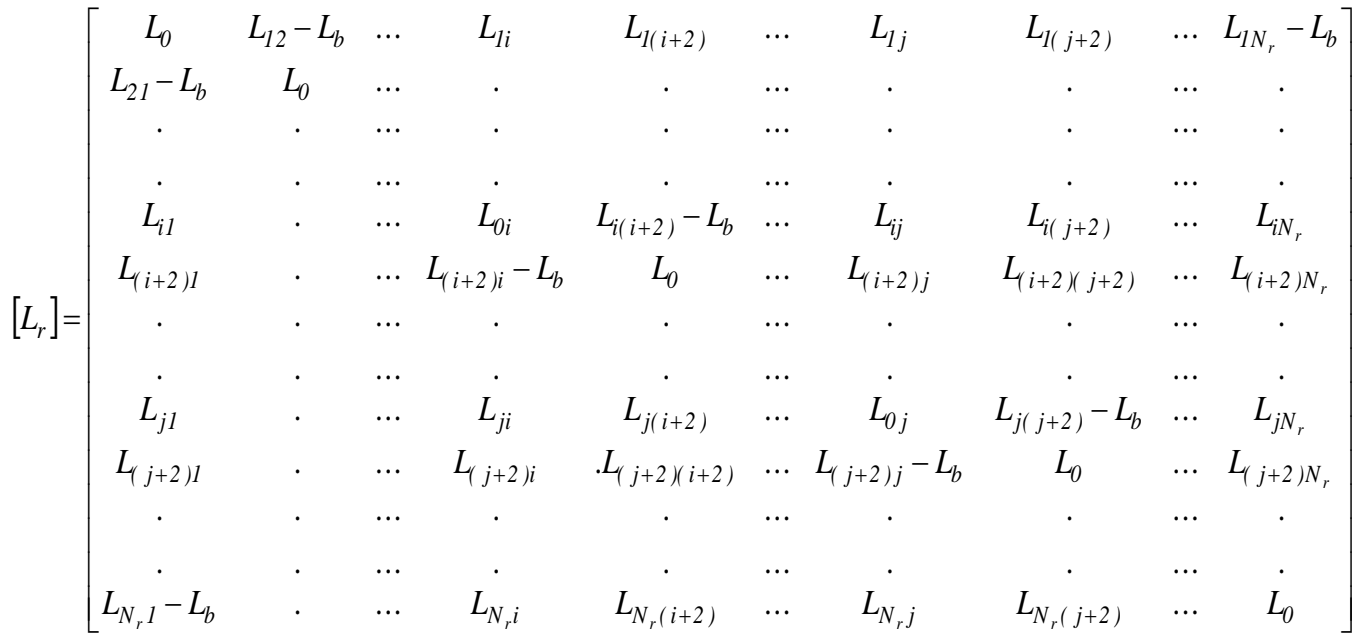

The matrix of the rotor resistances is written:

$$
\left[R_{r}\right]=\left[\begin{array}{cccccccccc}
R_{0} & -R_{b} & \ldots & 0 & 0 & \ldots & 0 & 0 & \ldots & -R_{b} \\
-R_{b} & R_{0} & \ldots & . & . & \ldots & . & . & \ldots & . \\
. & . & \ldots & . & . & \ldots & . & . & \ldots & . \\
. & . & \ldots & R_{0 i} & -R_{b} & \ldots & . & . & \ldots & . \\
. & . & \ldots & -R_{b} & R_{0} & \ldots & . & . & \ldots & . \\
. & . & \ldots & . & . & \ldots & . & . & \ldots & . \\
. & . & \ldots & . & . & \ldots & . & . & \ldots & . \\
0 & . & \ldots & . & . & \ldots & R_{0 j} & -R_{b} & \ldots & 0 \\
0 & . & \ldots & . & . & \ldots & -R_{b} & R_{0} & \ldots & 0 \\
. & . & \ldots & . & . & \ldots & . & . & \ldots & -R_{b} \\
-R_{b} & 0 & \ldots & . & . & \ldots & 0 & 0 & \ldots & R_{0}
\end{array}\right]
$$

Such as:

$R_{0 i}=2\left(R_{b}+2 R_{e}\right)$

$L_{0 i}=L_{i i}+2\left(L_{b}+2 L_{e}\right)$

$R_{0 j}=2\left(R_{b}+2 R_{e}\right)$

$L_{0 j}=L_{j j}+2\left(L_{b}+2 L_{e}\right)$

There is a difference between the two models of equations of a cage rotor with two broken bars, in the first model the two bars are adjacent and in the second are not adjacent. This difference appears particularly at resistance, self and mutual inductances of the relevant mesh breaking bars and the mutual inductance between them and the stator windings.

\section{PARAMETER DETERMINATION FOR ROTOR WITH SEVERAL BROKEN BARS}

The suggested approach was tested on a $4 \mathrm{~kW}, 2$ pole pairs, 28 bars squirrel cage induction generator which detailed parameters are given in Appendix.

The defects are introduced into the program by removing the blocks defining the bars concerned with the break, and by introducing new inductances and resistances calculated in the previous section. The variations of the equivalent resistance and the inductances of the rotor are given in table1.

TABLE I. PARAMETER VALUES FOR VARIOUS BROKEN ROTOR BARS

\begin{tabular}{|l|l|l|l|l|}
\hline \multirow{2}{*}{$\begin{array}{l}\text { Number of } \\
\text { BRB }\end{array}$} & \multicolumn{4}{|l|}{} \\
\cline { 2 - 5 } & $\boldsymbol{L}_{i i}(\boldsymbol{\mu H})$ & $\boldsymbol{L}_{0 i}(\boldsymbol{\mu H})$ & $\boldsymbol{L}_{k i}(\boldsymbol{\mu H})$ & $\boldsymbol{R}_{\boldsymbol{i}}(\boldsymbol{\mu} \boldsymbol{\Omega})$ \\
\hline 0 & 8.15 & 8.78 & -0.3 & 203.88 \\
\hline 1 & 16 & 16.7 & -0.6 & 213.88 \\
\hline 3 & 23.84 & 24.61 & -0.9 & 223.88 \\
\hline 4 & 31.68 & 32.52 & -1.2 & 233.88 \\
\hline 5 & 39.53 & 40.45 & -1.5 & 243.88 \\
\hline & 47.38 & 48.37 & -1.8 & 253.88 \\
\hline
\end{tabular}




\begin{tabular}{|l|l|l|l|l|}
\hline \multirow{2}{*}{$\begin{array}{l}\text { Number of } \\
\text { BRB }\end{array}$} & \multicolumn{4}{|l|}{} \\
\cline { 2 - 5 } & $\boldsymbol{L}_{i i}(\boldsymbol{\mu H})$ & $\boldsymbol{L}_{0 i}(\boldsymbol{\mu H})$ & $\boldsymbol{L}_{k i}(\boldsymbol{\mu H})$ & $\boldsymbol{R}_{0 i}(\boldsymbol{\mu} \boldsymbol{\Omega})$ \\
\hline 6 & 55.22 & 56.28 & -2.1 & 263.88 \\
\hline 7 & 63.07 & 64.20 & -2.4 & 273.88 \\
\hline 8 & 70.91 & 72.11 & -2.7 & 283.88 \\
\hline 9 & 78.76 & 80.04 & -3 & 293.88 \\
\hline 10 & 86.61 & 87.96 & -3.3 & 303.88 \\
\hline
\end{tabular}

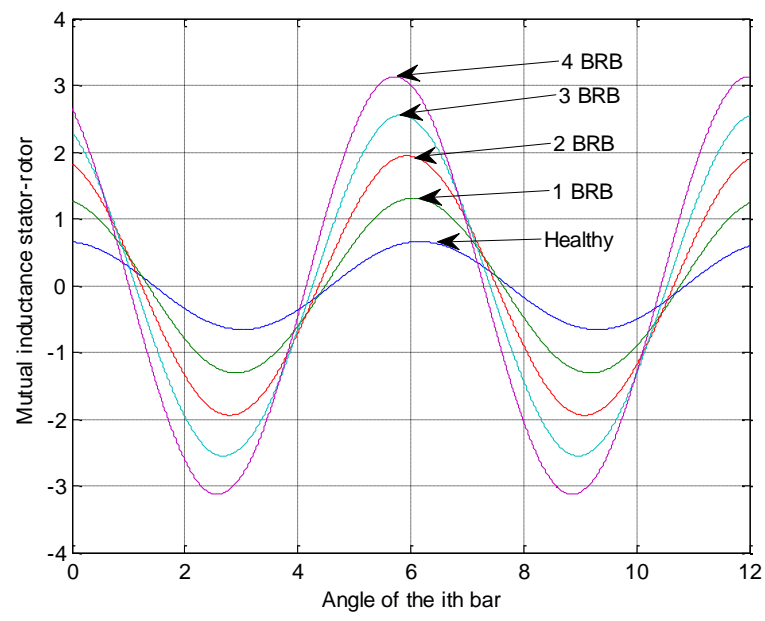

Fig. 9. Mutual inductance between $a$ stator phase and $i^{\text {th }}$ rotor loop

\section{CONCLUSION}

A generalized model of the squirrel-cage rotor in induction generator has been developed at the base of mathematical recurrences. The determination of leakage and the mutual inductances of the rotor meshes is carried out by using the winding function theory.

This model allows for the introduction of the defects and knowledge of the real currents in the rotor bars like in the end ring; what is difficult to realize by the 3-phase model.

One can determine analytical knowledge of the machine to produce quantifiable and analytical information.

The advantage of this model is that it is applicable to cage rotors with non-integral number of rotor bars per pole pair. The proposed approach can be used also for the simulation of variable speed induction machine drives under rotor faults.

\section{APPENDIX}

The parameters of our machines are illustrated in the following table:

TABLE II. INDUCTION GENERATOR PARAMETERS

\begin{tabular}{|l|c|c|}
\hline \multicolumn{1}{|c|}{ Parameters } & Symbols & Values \\
\hline Rated power & $P$ & $4 \mathrm{~kW}$ \\
Rated voltage & $V$ & $220 / 380 \mathrm{~V}$ \\
Rated current & $I$ & $15.2 / 8.8 \mathrm{~A}$ \\
Rated speed & $\Omega$ & $1465 \mathrm{rpm}$ \\
Pole-pair number & $p$ & 2 \\
Supply frequency & $f_{s}$ & $50 \mathrm{~Hz}$ \\
Power factor & $\cos \varphi$ & 0.83 \\
\hline
\end{tabular}

\begin{tabular}{|l|c|c|}
\hline \multicolumn{1}{|c|}{ Parameters } & Symbols & Values \\
\hline Rotor inertia & $J$ & $0.011 \mathrm{~kg} \cdot \mathrm{m}^{2}$ \\
Friction coefficient & $f$ & $8.73 \mathrm{e}^{-4} \mathrm{Nm} / \mathrm{rad} / \mathrm{s}$ \\
Stator phase turns in series & $N_{s}$ & 156 \\
Stator phase resistance & $R_{s}$ & $1.5 \Omega$ \\
Stator phase leakage inductance & $L_{l s}$ & $7 \mathrm{mH}$ \\
Stator magnetizing inductance & $L_{m s}$ & $0.55 \mathrm{H}$ \\
Rotor bar resistance & $R_{b}$ & $96.94 \mu \Omega$ \\
Rotor bar inductance & $L_{b}$ & $0.28 \mu \mathrm{H}$ \\
End-ring segment resistance & $R_{e}$ & $5 \mu \Omega$ \\
End-ring segment inductance & $L_{e}$ & $0.036 \mu \mathrm{H}$ \\
Number of rotor bars & $N_{r}$ & 28 \\
Mean radius air-gap & $r$ & $54 \mathrm{~mm}$ \\
Effective air-gap & $g$ & $0.28 \mathrm{~mm}$ \\
Length of air-gap & $l$ & $120 \mathrm{~mm}$ \\
& & \\
\hline
\end{tabular}

REFERENCES

[1] M.E.H Benbouzid. Bibliography on induction motor faults detection and diagnosis. IEEE Trans.On Energy Conversion, vol.14 , pp.1065-1074, Dec.1999

[2] Kumar, R.S. ; VIT Univ., Vellore, India ; Manimozhi, M. ; Enosh, M.T. A survey of fault detection and isolation in wind turbine drives. International Conference on Power, Energy and Control (ICPEC), 6-8 Feb. 2013, pp. $648-652$

[3] S. Williamson and M. J. Rabinson. Calculation of cage induction motor equivalent circuit parameters using finite elements," IEE Proceedings-B, vol. 138, No. 5, pp. 263-276, Sep. 1991.

[4] J. F. Bangura, Povinelli R.J., N.A.O Demerdash, R.H. Brown. Diagnostics of eccentricities and bars/end-ring connectors breakage in polyphase induction motors trough a combination of time series data mining and time-stepping coupled FE state space techniques. IEEE Trans. On Industry Applications, vol.39, N ${ }^{\circ} 4$, pp.1005-1013, July/August 2003.

[5] Meshgin - Kelk, H. Milimonfared, J. and Toliyat H.A. Interbar Currents and Axial Fluxes in Healthy and Faulty Induction Motors. IEEE Trans. On Industry Applications, vol.40, N¹, pp. 128-134, Jan./Feb 2004.

[6] H.A. Toliyat and T. A. Lipo. Transient analysis of cage induction machines under stator, rotor bar and end ring faults. IEEE Trans. On Energy Conversion, vol.10, n², pp.241-247, June 1995.

[7] Vinod V. Thomas, Krishna Vasudevan, and V. Jagadeesh Kumar. Online cage rotor fault detection using air-gap torque spectra. IEEE Trans. On Energy Conversion, vol. 18, N² 2, pp.265-270, June 2003.

[8] K. R. Cho, J.H. Lang, and S.D. Umans. Detection of broken rotor bars in induction motors using state and parameter estimation. IEEE Trans. On Industry Appl., pp.702-709, vol.28, N³, May/June 1992.

[9] F. Filippetti, G. Franceshini, C. Tassoni, and P. Vas. Broken bar detection in induction machines: Comparison between current spectrum approach and parameter estimation approach. IEEE-IAS, Annual Meeting Conf., pp.95-102, vol.2, Denver USA,1994.

[10] H. R. Fudeh and Ong C. M. Modeling and analysis of induction machines containing space harmonics. Pt. I, II and III. IEEE Trans. On Power Apparatus and Systems, vol.PAS-102, nº, pp.2608-2628, Aug. 1985

[11] Alfredo R. Muñoz and Thomas Lipo. Complex vector model of the squirrel cage inductance machine including instantaneous rotor bar currents. IEEE Trans. On Industry Applications, vol.35, nº, pp.13321340, 1999.

[12] Omar, T. ; Lahcene, N. ; Rachid, I. ; Maurice, F. Modeling of the induction machine for the diagnosis of rotor defects. Part I. An approach of magnetically coupled multiple circuits. IECON 2005. 31st Annual Conference of IEEE Industrial Electronics Society, 2005.

[13] Issam Attoui , Amar Omeiri. Modeling, control and fault diagnosis of an isolated wind energy conversion system with a self-excited induction generator subject to electrical faults. Energy Conversion and Management 82 (2014) pp. 11-26

[14] Xiaogang Luo, Yuefeng Liao, and H.A. Toliyat. Multiple coupled circuit modeling of induction machines. IEEE Trans. On Industry Applications, vol.31, $\quad \mathrm{N}^{\circ} 2, \quad$ pp.311318, $\quad$ March/April 1995. 